\title{
ANÁLISIS BIBLIOGRÁFICO SOBRE EL SENTIMIENTO DE MIEDO DURANTE LA PANDEMIA DE LA COVID-19 EN PERSONAS MAYORES
}

\author{
Nieves Gutiérrez Ángel \\ Universidad de Almería \\ Nga212@ual.es \\ Isabel Mercader Rubio \\ Universidad de Almería \\ Imercade@ual.es \\ David Padilla Góngora \\ Universidad de Almería \\ Dpadilla@ual.es
}

Recepción Artículo: 18 mayo 2021

Admisión Evaluación: 18 mayo 2021

Informe Evaluador 1: 23 mayo 2021

Informe Evaluador 2: 25 mayo 2021

Aprobación Publicación: 01 junio 2021

\section{RESUMEN}

Introducción: En las últimas encuestas sobre la población española, la tasa de personas mayores con más de 65 años gira entorno al 19\% de la población. Debido a la situación provocada por la pandemia de la COVID19, se establecieron distintas medidas de carácter social y sanitarias, entre ellas el confinamiento domiciliario, el distanciamiento social, o el cierre de comercios y escuelas. Ante estas situaciones, las personas pueden desarrollar conductas de escape o evitación debido a la falta de control y al sentimiento de amenaza. Objetivo: El objetivo de este trabajo es analizar las publicaciones en la literatura científica sobre aquellos trabajos que abordan el sentimiento de miedo en personas mayores durante la duración de la pandemia de la COVID-19. Metodología: Para la búsqueda de la información se utilizaron las siguientes bases de datos: Dialnet, Scopus, Psicodoc, Psycinfo, Eric y WOS. Empleando como descriptores: "afraid" and "COVID" and "aged". Se escogieron aquellas publicaciones que tenían a su disposición el texto completo, su idioma de publicación fuese inglés, portugués o español, y su fecha de publicación estuviera comprendida entre los años 2020 y 2021. Resultados: Los resultados encontrados mencionan los sentimientos relacionados con el miedo al contagio, a estar en lugares públicos y a salir de casa. Conclusiones: Este trabajo confirma las consecuencias negativas a nivel emocional que está provocando la COVID-19.

Palabras clave: mayores; covid-19; consecuencias emocionales; miedo

\section{ABSTRACT}

Bibliographic analysis on the feeling of fear during the covid-19 pandemic in older people . Introduction: In the latest surveys on the Spanish population, the rate of older people over 65 years of age is 


\section{ANÁLISIS BIBLIOGRÁFICO SOBRE EL SENTIMIENTO DE MIEDO DURANTE LA PANDEMIA DE LA COVID-19 EN PERSONAS MAYORES}

around $19 \%$ of the population. Due to the situation caused by the COVID-19 pandemic, different social and health measures were established, including home confinement, social distancing, or the closure of shops and schools. In these situations, people can develop escape or avoidance behaviors due to a lack of control and a feeling of threat. Objective: The objective of this work is to analyze the publications in the scientific literature on those works that address the feeling of fear in older people during the duration of the COVID-19 pandemic. Methodology: The following databases were used to search the information: Dialnet, Scopus, Psicodoc, Psycinfo, Eric and WOS. Using as descriptors: "afraid" and "COVID" and "aged". Those publications were chosen that had the full text at their disposal, their language of publication was English, Portuguese or Spanish, and their publication date was between 2020 and 2021. Results: The results found mention the feelings related to the fear of contagion, being in public places and leaving the house. Conclusions: This work confirms the negative emotional consequences that COVID-19 is causing.

Keywords: elderly; covid-19; emotional consequences; fear

\section{INTRODUCCIÓN}

Según la Organización Mundial de la Salud (OMS, 2020) el coronavirus (COVID-19) ha causado más de 383.000 muertes en todo el mundo. En el caso de nuestro país, España, es el tercer país del mundo en cuanto a casos durante el mes de marzo de 2020, hecho que lleva a tomar distintas medidas de carácter social y sanitarias ante dicha situación. Entre ellas, el confinamiento domiciliario, el distanciamiento social, o el cierre de comercios y escuelas (León, 2021). Medidas todas ellas, que, si bien tenían como finalidad parar el avance y contagio del virus, no debemos obviar que han provocado consecuencias negativas en torno al estado psicológico de la población, principalmente relacionados con el miedo, la ansiedad, el estrés, la depresión, trastornos del sueño, o irascibilidad (Huang et al., 2020; Serafini, 2020; Wang, Zhang, Zhao, Zhang yJiang, 2020).

En este sentido, dichas medidas, aunque con carácter sanitario y preventivo, dieron lugar al aislamiento, situación especialmente delicada en las personas mayores y en el contexto de nuestro país, puesto que el envejecimiento supone en España un hecho evidente y característico de nuestra sociedad, donde el número de personas que pertenecen a este colectivo se ha incrementado de manera exponencial (Montoya et al., 2018). Tanto es así que a día de hoy, la tasa de personas mayores con más de 65 años gira entorno al 19\% de la población (Fernández-Ballesteros y Sánchez-Izquierdo, 2020).

En este trabajo nos vamos a centrar en el sentimiento del miedo principalmente. Para Quezada (2020) el miedo es una emoción básica ante una situación que percibimos como amenazante para nuestra integridad, que lleva asociada una respuesta adaptativa en nuestro comportamiento (Wodjat y Pape, 2013).

Aunando ambos conceptos, podemos afirmar que la COVID-19 supone una amenaza en la que además se unen la incertidumbre que acompaña a la situación social y sanitaria, dando lugar a una situación que se percibe como potencialmente peligrosa, impredecible e incontrolable (Quezada, 2020).

En relación con estas ideas, tenemos que tener en cuenta que ante estos hechos las personas pueden desarrollar conductas de escape o evitación debido a la falta de control y el sentimiento de amenaza (Raines, Oglesby, Unruh, Capron \& Schmidt, 2013), 0 la incertidumbre (Flores, López, Cobos, \& Vervliet, 2018a, 2018b).

De tal manera, este estudio se enmarca dentro de aquellos que han intentado averiguar las consecuencias negativas a nivel emocional que ha tenido la COVID-19 (Brooks et al., 2020; Cao et al., 2020; Gao et al., 2020; Liu et al., 2020; Roy et al., 2020), pero en nuestro caso, tomando como muestra a personas mayores.

\section{OBJETIVOS DE LA INVESTIGACIÓN}

El objetivo de este trabajo es analizar las publicaciones en la literatura específica sobre aquellos trabajos que abordan el sentimiento de miedo en personas mayores durante la duración de la pandemia de la COVID-19.

\section{METODOLOGÍA Y/O INSTRUMENTOS UTILIZADOS}

El método denominado revisión bibliográfica ha sido empleado para investigar y sintetizar todos aquellos hallazgos que en el último año han sido publicados acerca del miedo sufrido durante la COVID-19 en personas 
mayores.

Esta modalidad, se basa en realizar una investigación documental, es decir, recopilar información ya existente sobre un tema o problema, siendo el tema elegido sobre la pandemia o COVID-19 y el sentimiento de miedo que produce en las personas mayores

Para ello, aplicamos un proceso que se divide en cuatro fases:

FASE 1: Búsqueda

Se realizó una búsqueda entre los años 2020 y 2021. Empleando como descriptores "afraid" and "COVID" and "aged". Además, se utilizaron algunas de las indicaciones aportadas por Cooper y Hedges (1994) y Cooper (2009) tales como: revistas revisadas por pares), y referencia bases de datos e índices de citas.

FASE 2: Proceso de selección

Sobre estas ideas, las bases de datos empleadas fueron WOS, Scopus, Dialnet, Psicodoc, Psycinfo y Eric. Aplicando como filtros para la búsqueda estuviera a disposición el texto completo, y su idioma de publicación fuese inglés, portugués o español.

FASE 3: Análisis metodológico

Para escoger los artículos se siguió la Declaración PRISMA (Moher et al., 2009). De tal manera, inicialmente se encontraron 60 artículos en dicha búsqueda. Tras ello, se procedió a eliminar los duplicados y a revisar tanto el título, como el resumen de cada uno de ellos. Finalmente, el número de trabajos se redujo a 6, los cuales cumplían los siguientes criterios:

1.Son publicaciones que están escritas en español, inglés o portugués comprendidas entre los años 2020 y 2021.

2.Son trabajos publicados en revistas que son revisadas por pares y son estudios empíricos.

3.Abordan el tema de las consecuencias psicológicas de la COVID, y en concreto del sentimiento de miedo

4. Los participantes son personas mayores.

FASE 4: Síntesis y aportaciones principales de los resultados de la búsqueda.

FIGURA 1. Diagrama de flujo PRISMA de selección de estudios. PRISMA, elementos de informe preferidos para revisiones sistemáticas y metaanálisis. Fuente. Basado en Moher et al. (2009).

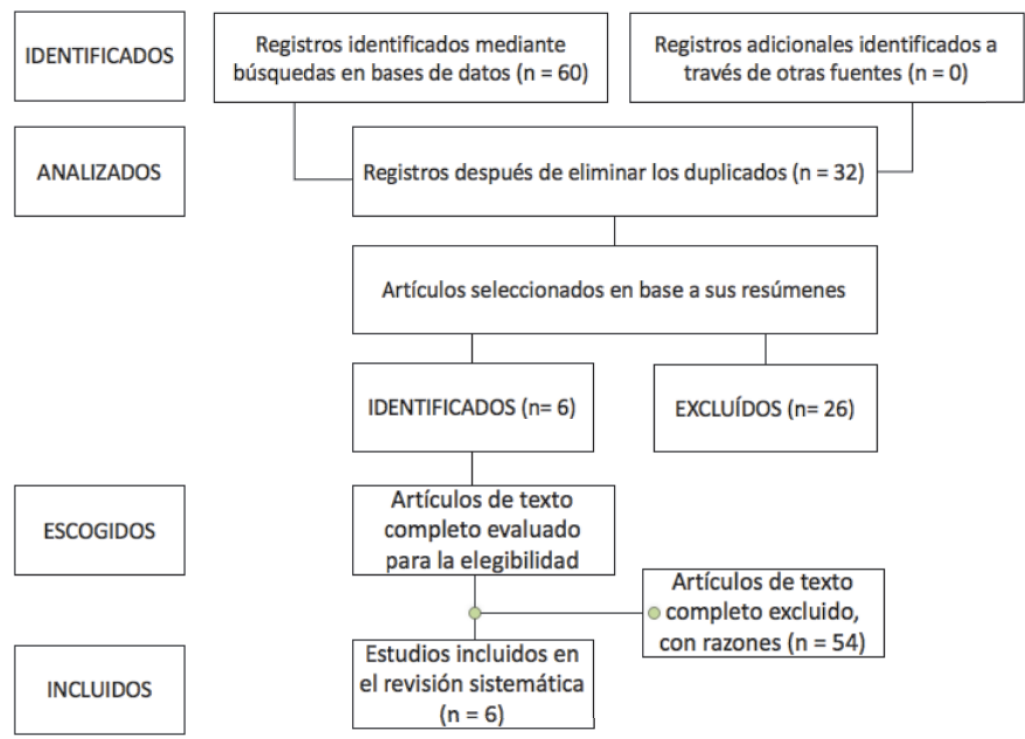




\section{ANÁLISIS BIBLIOGRÁFICO SOBRE EL SENTIMIENTO DE MIEDO DURANTE LA PANDEMIA DE LA COVID-19 EN PERSONAS MAYORES}

\section{RESULTADOS ALCANZADOS}

Los resultados obtenidos ponen de manifiesto el hecho de que sentir miedo es una de las emociones más comunes que están padeciendo las personas mayores durante la epidemia. Todo esto, se une a la aparición de sentimientos relacionados con el nerviosismo, la culpa o la tristeza.

En este sentido, el principal temor que poseen las personas mayores se relaciona con el contagio y con la muerte ante la COVID-19 (Kowalczuk y G bski, 2021; Ortiz et al., 2020; Sandín et al., 2020). Y ante el mismo, la muestra adopta como medida seguir fielmente las normas, encontrando una relación directa y positiva entre el miedo a contraer COVID-19 y el cumplimiento con las reglas de aislamiento (Kowalczuk y G bski, 2021). Este miedo además lo poseen tanto de forma propia e individual, como hacia las personas de su entorno, ya sean familiares o conocidos, ante lo que el contagio o la muerte de las personas cercanas también se convierte en un miedo.

Se evidencia que también se encuentra, el miedo a los lugares públicos. En este sentido, el estudio elaborado por Fabisiak et al. (2020) muestra el hecho de que alrededor del 15\% de las personas mayores tienen miedo de estar o utilizar lugares públicos, siendo más frecuente este miedo en mujeres, que en hombres. Ante este hecho, además la muestra que compuso el estudio, manifestó en un 90\% preferir estar en espacios abiertos (como parques, paseos, bulevares, plazas, etc.). Resultados similares a los aportados por Sandín et al. (2020), quienes demuestran en su estudio el miedo de las personas mayores a salir de casa y al aislamiento social.

Siguiendo a estos mismo autores, también establecen como resultados la aparición de otros miedos en menor orden de frecuencia, relacionados con no poder ver a los familiares, y no poder celebrar actos importantes. A lo que se suma el sentimiento de haber perdido el contacto directo con personas cercanas, así como de sus actividades cotidianas (Fabisiak et al., 2020).

Los hallazgos en otros estudios, como el de Tsugawa et al. (2020), toman como muestra a personas mayores con Azheimer, aportando como principal resultado el desconocimiento de la gravedad de la situación provocada por la pandemia.

A pesar de todos estos resultados negativos, el estudio elaborado por Sandín et al. (2020), también dedica un apartado a evaluar cuáles son los efectos positivos que la COVID-19 ha tenido en la población mayor. En este sentido destaca el valor que la muestra otorga a cuestiones relacionadas con poder salir a la calle o la realización de actividades al aire libre. La importancia que conceden a las relaciones interpersonales y el sentimiento de querer compartir más tiempo con la familia.

\section{DISCUSIÓN}

El objetivo de este trabajo fue analizar las publicaciones en la literatura específica sobre aquellos trabajos que abordan el sentimiento de miedo en personas mayores durante la duración de la pandemia de la COVID-19.

Los resultados encontrados confirman el hecho, también señalado en otros estudios, de las consecuencias negativas a nivel emocional que está teniendo la COVID-19 (Brooks et al., 2020; Cao et al., 2020; Gao et al., 2020; Liu et al., 2020; Roy et al., 2020). En cuanto al sentimiento de miedo, todos los resultados encontrados destacan el haber padecido el miedo al contagio y a la muerte como el más prevalente (Kowalczuk y G bski, 2021; Ortiz et al., 2020; Sandín et al., 2020).

Siendo el miedo a estar en lugares públicos y a salir de casa el dominante (Sandín et al., 2020). Mientras que, el menos prevalente, es el miedo relacionado con no poder ver a los familiares, y no poder celebrar actos importantes (Fabisiak et al., 2020).

En definitiva, se hace patente la existencia de emociones negativas asociadas a la situación que venimos describiendo, y los resultados-evidencian como junto al miedo también aparecen otras emociones negativas relacionadas con sentirse ansioso, sufrir depresión o incluso sentimientos relacionados con la irritación.

\section{CONCLUSIONES}

Podemos concluir este trabajo, afirmando que el miedo que experimentan las personas mayores se ejerce desde dos planos o visiones: La primera de ella, relacionada con el miedo individual y propio ante el contagio 0 
incluso la propia muerte, y, la segunda, desde un punto de vista mucho más global, que también implica un miedo de carácter más social, como es el miedo a salir de casa, o a permanecer en un lugar público.

Al respecto, las actitudes principales de las personas mayores ante dicha situación se han basado principalmente en la prevención ante un posible contagio, estar muy informados con respecto a la situación social y sanitaria, una alta comprensión de la gravedad de la situación y de sus consecuencias sanitarias, y un cumplimiento estricto de las medidas tomadas (Fernández-Ballesteros y Sánchez-Izquierdo, 2020).

Sin embargo, pese a la situación tan alarmante que sufre España con respecto a la COVID-19, Ios trabajos de estos autores muestran como las personas mayores manifiestan estar más preocupadas por otros temas de índole social, como la política o la economía, que por la enfermedad.

\section{REFERENCIAS BIBLIOGRÁFICAS}

Brooks, S. K., Webster, R. K., Smith, L. E., Woodland, L., Wessely, S., Greenberg, N. \& Rubin, G. J. (2020). The psychological impact of quarantine and how to reduce it: Rapid review of the evidence. Lancet, 395, 912-920. https://doi.org/10.1016/S0140-6736(20)30460-8.

Cao, W., Fang, Z., Hou, G., Han, M., Xu, X., Dong, J. \& Zheng, J. (2020). The psychological impact of the COVID19 epidemic on college students in China. Psychiatry Research, 11, 29-34. https://doi.org/10.1016/j.psychres.2020.112934.

Cooper, H. (2009). Research Synthesis andMeta-Analysis: A Step-By-Step Approach. Sage.

Cooper, H. y Hedges, L. V. (1994). The Handbook of Research Synthesis. Russell Sage.

Fabisiak, B., Jankowska, A. \& Kłos, R. (2020). Attitudes of Polish Seniors toward the Use of Public Space during the First Wave of the COVID-19 Pandemic. International Journal of Environmental Research and Public Health, 17(23), 1-27.

Fernández-Ballesteros, R. \& Sánchez-Izquierdo Alonso, M. (2020). Impacto del COVID-19 en personas mayores en España: algunos resultados y reflexiones. Clínica y Salud, 31(3), 165-169.

Flores, A., López, F. J., Vervliet, B. y Cobos, P. L. (2018a). Dime si toleras la incertidumbre y te dire si evitas demasiado. Ciencia Cognitiva, 12(3), 60-73.

Flores, A., López, F. J., Vervliet, B. y Cobos, P. L. (2018b). Intolerance of uncertainty as a vulnerability factor for excessive and inflexible avoidance behavior. Behaviour Research and Therapy, 104, 34-43. https://doi.org/10.1016/j.brat.2018.02.008.

Gao, J., Zheng, P., Jia, Y., Chen, H., Mao, Y., Chen, S. .. \& Dai, J. (2020). Mental health problems and social media exposure during COVID-19 outbreak. Plos One, 15, e0231924. https://doi.org/10.1371/journal.pone.0231924.

Huang,C., Wang,Y., Li,X., Ren,L., Zhao,J., Hu,Y. ...CaoB.(2020) Clinical features of patients infected with 2019 novel coronavirus in Wuhan, China. The Lancet, 395(10223), 497-506. doi: 10.1016 / S0140-6736 (20) 30183-5

Kowalczuk, I. \& G bski, J. (2021). Impact of Fear of Contracting COVID-19 and Complying with the Rules of Isolation on Nutritional Behaviors of Polish Adults. International Journal of Environmental Research and Public Health, 18(4), 1-12.

León, M. I. G. (2021). Disminución de la ansiedad en las víctimas del bullying durante el confinamiento por el COVID-19. Revista De Educación a Distancia (RED), 21(65), 1-20.

Liu, N., Zhang, F., Wei, C., Jia, Y., Shang, Z., Sun, L. ... \& Liu, W. (2020). Prevalence and predictors of PTSS during COVID-19 outbreak in China hardest-hit areas: Gender differences matter. Psychiatry Research, 112921. doi: https://doi.org/10.1016/j.psychres.2020.112921.

Moher, D., Liberati, A., Tetzlaff, J., Altman, D. G. y the PRISMA Group (2009). Preferred reporting items for systematic reviews and meta-analyses: the PRISMA statement. PLOS Med. 6, e1000097. doi: 10.1371/journal.pmed.1000097 


\section{ANÁLISIS BIBLIOGRÁFICO SOBRE EL SENTIMIENTO DE MIEDO DURANTE \\ LA PANDEMIA DE LA COVID-19 EN PERSONAS MAYORES}

Montoya, A. M., Parra, J. M. A., Góngora, D. P., Pérez, P. R., \& Liria, R. L. (2018). Consumo farmacológico y salud en personas mayores. Revista INFAD de Psicología. International Journal of Developmental and Educational Psychology, 4(1), 235-240.

Ortiz, D. D. L. Á. C. \& Arboleda, J. S. J. (2020). Actividad fisiológica, alimenticia y psicológica de los adultos mayores durante la cuarentena del Covid-19 en el Centro de Atención Integral (CEAM) la Delicia. Quito, Ecuador. Revista Científica de FAREM-Esteli: Medio Ambiente, Tecnología y Desarrollo Humano, 35, 88-108.

Quezada, V. E. (2020). Miedo y psicopatología la amenaza que oculta el Covid-19. Cuadernos de Neuropsicología, 14(1), 19-23.

Raines, A. M., Oglesby, M. E., Unruh, A. S., Capron, D. W. y Schmidt, N. B. (2013). Perceived control: A general psychological vulnerability factor for hoarding. Personality and individual differences, 56, 175-179. doi: 10.1016/j.paid.2013.09.005

Roy, D., Tripathy, S., Kar, S. K., Sharma, N., Verma, S. K. \& Kaushal, V. (2020). Study of knowledge, attitude, anxiety \& perceived mental healthcare need in Indian population during COVID-19 pandemic. Asian Journal of Psychiatry, 102083. doi: 10.1016/j.ajp.2020.102083.

Sandín, B., Valiente, R. M., García-Escalera, J. \& Chorot, P. (2020). Impacto psicológico de la pandemia de COVID-19: Efectos negativos y positivos en población española asociados al periodo de confinamiento nacional. Revista de Psicopatología y Psicología Clínica, 25(1), 1-22.

Serafini, G., Parmigiani, B., Amerio, A., Aguglia, A., Sher, L. \& Amore, M. (2020). The psychological impact of COVID-19 on the mental health in the general population. QJM: An International Journal of Medicine, 113(8), 531-537.

Tsugawa, A., Sakurai, S., Inagawa, Y., Hirose, D., Kaneko, Y., Ogawa, Y. ... \& Shimizu, S. (2020). Awareness of the COVID-19 Outbreak and Resultant Depressive Tendencies in Patients with Severe Alzheimer's Disease. Journal of Alzheimer's Disease, (Preprint), 1-3.

Wang, G., Zhang, Y., Zhao, J., Zhang, J. yJiang, F. (2020). Mitigate the effects of home confinement on children during the COVID-19 outbreak. Lancet (London, England), 395(10228), 945-947. https://doi.org/10.1016/S0140-6736(20)30547-X

Wotjak, C. T. \& Pape, H.-C. (2013). Neuronal circuits of fear memory and fear extinction. E-Neuroforum, 19(3), 47-56. https://doi.org/10.1007/s13295-013-0046-0 